\title{
Sensitivity Enhancement of a Graphene, Zinc Sulfide-based Surface Plasmon Resonance Biosensor With an Ag Metal Configuration in the Visible Region
}

Bhishma Karki ( $\nabla$ magnum.photon@gmail.com )

Tribhuvan University - Trichandra Multiple Campus https://orcid.org/0000-0003-4351-8099

Arun Uniyal

DIT University

Brajlata Chauhan2

DIT: DIT University

Amrindra Pal

DIT University

\section{Research Article}

Keywords: Surface plasmon resonance, Zinc Sulfide, Sensitivity, Reflectance, Analyte

Posted Date: October 4th, 2021

DOl: https://doi.org/10.21203/rs.3.rs-854012/v1

License: (c) (i) This work is licensed under a Creative Commons Attribution 4.0 International License.

Read Full License

Version of Record: A version of this preprint was published at Journal of Computational Electronics on January 14th, 2022. See the published version at https://doi.org/10.1007/s10825-022-01854-4. 


\title{
Sensitivity Enhancement of a Graphene, Zinc Sulfide-based surface plasmon resonance biosensor with an Ag metal configuration in the visible region
}

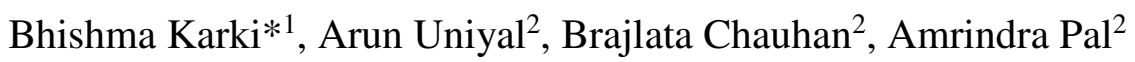 \\ ${ }^{1}$ Department of Physics, Tri-Chandra Multiple Campus, Tribhuvan University, Kathmandu-44600, Nepal \\ ${ }^{2}$ Department of ECE, DIT University, Dehradun-248009, India \\ *Corresponding Author: Email address: magnum.photon@gmail.com
}

\begin{abstract}
A biosensor based on the modified Kretschmann configuration is proposed here. The sensitivity of the conventional prism-based sensor using angular interrogation is low. To enhance the sensor's performance, layers of zinc sulfide $(\mathrm{ZnS})$ and graphene have been deposited over the metal layer. The angular interrogation technique is used to analyze the performance of the sensor. The thickness of the Ag metal has been optimized. The thickness of the Ag metal is taken as $50 \mathrm{~nm}$ because minimum reflectance has been achieved. With the combinations of the four layers of $\mathrm{ZnS}$ and one graphene layer, the maximum sensitivity attained is $305^{\circ} / R I U$. Performance parameters such as detection accuracy, FWHM, and quality factor of the sensor have been evaluated as obtained as $0.33 \mathrm{deg}^{-1}, 3.05 \mathrm{deg}, 100.7 \mathrm{RIU}^{-1}$, respectively. The proposed sensor has potential application in the field of biochemical and biological analyte detection.
\end{abstract}

Keywords: Surface plasmon resonance, Zinc Sulfide, Sensitivity, Reflectance, Analyte

\section{Introduction}

The concept of Surface plasmon resonance (SPR) gains popularity from the last two decades in biosensing applications. The critical factor for the success of SPR sensor technology is its exclusive properties like sensing abilities, reliability, accuracy, compatibility, and label-free detection measurements [1]-[4]. Besides this, food safety, environmental and gas detection [5], and many physical, chemical, and biological quantities measurements are easily possible using the SPR based biosensor [6], [7]. The phenomenon of SPR is oscillations of free electrons named surface plasmons (SPs) or surface plasmon polaritons (SPP) at metal-dielectric boundary [8], [9]. SPs are electromagnetic waves propagating at the metal boundary. To excite the SPs at the metal-dielectric interface, a coupling prism is preferred to excite the momentum of input TM or $\mathrm{P}$ polarized radiation to match the momentum of surface plasmon wave (SPW) [10]. Achieving this condition of phase matching between Surface plasmon wave vector (SPWV) and Evanescent 
wave vector (EWV) of input wave [11], the maximum energy shifts from input wave to SPW [8], [9]. A sharp dip in the resonance curve is experienced at the resonance condition concerning the sensor's dielectric and metal layer properties [12]. Two types of prism coupling are used in SPR sensor designs, namely Otto [13] and Kretschmann [14] based structures. With ease in the implementation, Kretschmann, design type is generally preferred. The angle interrogation technique is used with monochromatic input radiation to generate surface plasmons (SPs) [12]. The advantage of choosing angle interrogation with the Kretschmann configuration provides the high signal-to-noise ratio (SNR) using a single source of input radiation [15], [16]. Gold (Au), silver $(\mathrm{Ag})$, copper $(\mathrm{Cu})$ and, Aluminum $(\mathrm{Al})$ is preferred as metals used in SPR sensors. However, each metal has its own merits with demerits [17]. Au is preferred mainly because of its optical properties, such as it is highly resistive to oxidation and corrosion, giving good chemical stability with low losses[18]. The demerit is that the adhesion of the analyte with the gold metal layer is less, and a broader SPR curve is obtained due to this sensitivity of the sensor is affected. Silver (Ag) is mainly used because of its exclusive properties, like increasing sensitivity but susceptibility to oxidation. The metal oxidation may be reduced by using bimetallic layers [19] and it improving the sensor's sensitivity also. Silver has a narrower SPR curve dip compared to gold. The fact that single silver layer cannot increase the sensitivity significantly. The dielectric material (like 2-D materials, semiconductors) must be used for performance enhancement of the SPR biosensor such as the figure of merit (FoM), sensitivity [20] etc.

Zinc sulfide $(\mathrm{ZnS})$ is a compound semiconductor [21]. It has extraordinary optoelectronic properties, such as a wide energy bandgap $(3.72 \mathrm{eV} / 3.77 \mathrm{eV})$ for cubic zinc blende and hexagonal wurtzite (WZ). Its applications are in non-linear optical devices, lasers, gas sensors [21], [22], UV light sensors, etc., with good chemical and thermal stability [23]. These optoelectronic properties of the material make it a preferred choice for the proposed work. With the technological research advancement on 2-D materials, its inclusion in photonics, optics, electronics, plasmonic devices shows tremendous growth [24]. Graphene is a standard fundamental recognition element (BRE) used in sensor design. Experimentally it is found that graphene is a 2D material for biosensing application, enhance the sensitivity significantly. Graphene is an electroactive and transparent material [25]. It has various optical and electrical features [26], such as a large surface-to-volume ratio, ring-based crystal structure, zero-band gap, 
low cost, and highly conductive and elastic. The absorption rate which it offers makes it suitable for the biosensing field [26].

Various research groups proposed multilayer bio-sensor structures based on performance parameters enhancement such as sensitivity, FoM, detection accuracy, and full width half maximum (FWHM). Wu et al. [18] designed an SPR sensor; with the increase in the number of graphene layers, their sensor's sensitivity extended to $25 \%$. Ouyang et al. proposed a sensor with TMDCs materials and one silicon sheet to investigate the sensitivity. They achieved it as $155.68 \%$ RIU [27]. Gan et al. suggested a sensor design using silver as a metal layer and franckeite as 2-D material and reported the sensitivity of $188^{\circ} / R I U$ [28]. Pal and Jha implemented an SPR based sensor with barium titanate as 2D material with a bi-metallic silver layer, and sensitivity $280^{\circ} / R I U$ is obtained [11]. Srivastava and Prajapati demonstrated a new sensor design using silicon, blue phosphorene, $\mathrm{MoS}_{2}$ hybrid structure, and sensitivity $230.66^{\circ} /$ $R I U$ is found [29]. Singh et al. theoretically analyzed an SPR sensor design using graphene and black phosphorus and $\mathrm{Au}$ as a metal layer; for seven black phosphorus layers and single graphene layer, they achieved a sensitivity of $218^{\circ} / R I U$ [24]. In this work, a modified Kretschmann configuration-based SPR biosensor is proposed, consists of multilayers of Ag, $\mathrm{ZnS}$, Graphene, and sensing media placed over BK7 prism. A novel material, zinc sulfide, has been introduced to improve the sensor's sensitivity and other performance parameters also. The characteristics parameter of the proposed sensor is also compared with the conventional sensor's parameter. The performing parameters like sensitivity(S), Figure of merit (FoM), and detection accuracy (DA) are being evaluated.

The manuscript is structured as: Section 2 gives modeling of the proposed sensor along with mathematical expressions. Section 3, results and discussions, has been provided. Section 4 gives the conclusion of the proposed work.

\section{Mathematical expressions and theoretical analysis of proposed biosensor}

The proposed setup for the biosensor is shown below in figure 1. It is a four-layer model containing Ag metal, $\mathrm{ZnS}$, graphene, and a sensing medium. BK7 prism is used as a coupling prism. A $633 \mathrm{~nm}$ optical signal is incident at one face of the prism, and it is reflected and coming 
out from the other face. Here He/Ne laser source is used. The refractive index (RI) of the BK7 prism is 1.515 [24]. The RI of metal layers is computed using the Drude-Lorentz model:

$\mathrm{n}_{\text {metal }}^{2}=1-\frac{\lambda^{2} \lambda_{\mathrm{c}}}{\lambda_{\mathrm{p}}^{2}\left(\lambda_{\mathrm{c}}+\mathrm{i} \lambda\right)}$

The notations $\lambda_{\mathrm{c}}$ and $\lambda_{\mathrm{p}}$ indicates collision and plasma wavelengths, and their values are $\lambda_{\mathrm{c}}=$ $1.7614 \times 10^{-5} \mathrm{~m}$ and $\lambda_{\mathrm{p}}=1.4541 \times 10^{-7} \mathrm{~m}$, respectively [26]. A layer of $\mathrm{ZnS}$ (thickness $d_{2}$ ) is deposited over the $\mathrm{Ag}$ metal (thickness $d_{1}$ ). A layer of the graphene (thickness $d_{3}$ ) is deposited over the $\mathrm{ZnS}$. The sensing layer is joined with the graphene layer.

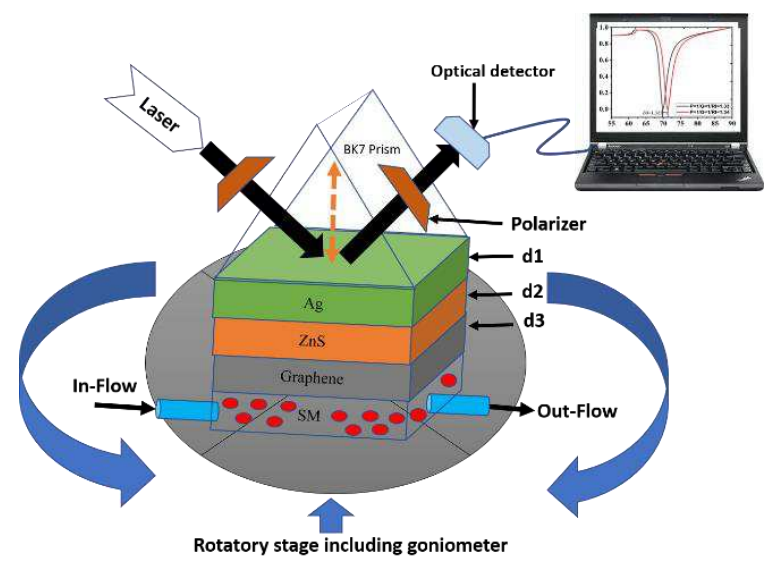

Figure 1: Proposed layered SPR biosensor design

Table 1: Materials and their width used in the proposed study

\begin{tabular}{|c|c|c|c|}
\hline Metal/dielectric layer & Width/thickness $(\mathbf{n m})$ & Refractive Index & Ref \\
\hline $\mathrm{Ag}$ & $45\left(D_{1}\right)$ & $0.0561+i * 4.276$ & {$[26]$} \\
\hline $\mathrm{ZnS}$ & $\mathrm{P} * 3 \mathrm{~nm}\left(D_{2}\right)$ & $2.35521+\mathrm{i} * 0.00523$ & {$[23]$} \\
\hline Graphene & $\mathrm{G} * 0.5 \mathrm{~nm}\left(D_{3}\right)$ & $3+1.1487 \mathrm{i}$ & {$[30]$} \\
\hline Sensing layer & & $133+\delta \mathrm{n}$ & \\
\hline
\end{tabular}

Table 1 contains the thickness parameters and refractive index of the used materials. $\delta n$ is the change in RI after bio/chemical reactions, and $\mathrm{P}$ and $\mathrm{G}$ indicate no. of $\mathrm{ZnS}$ and graphene layers. The combination of the evanescent wave from the input source and plasmons of the plasmonic layer gives surface plasmonic wave (SPW). It propagates on the metal and dielectric boundary, satisfying the resonance condition given by [23]:

$\mathrm{k}_{\mathrm{x}}=\mathrm{n}_{\mathrm{BK} 7} * \frac{\omega}{\mathrm{c}} * \sin \theta$ 
where $n_{\mathrm{BK} 7}$ RI of coupling prism, $\omega$ is the angular frequency, $c$ is the speed of light, $\theta$ is the resonance angle. The biosensor response analysis is done by the popular transfer matrix method (TMM), which does not consider approximation and gives accurate results. The calculations for reflectance by TMM are given here by characteristics matrix after applying tangential and electrical field component's boundaries.

$\mathrm{C}=\prod_{\mathrm{k}=2}^{\mathrm{n}-1} \mathrm{C}_{\mathrm{r}}$

where $\mathrm{C}_{\mathrm{r}}$ is $\mathrm{r}^{\text {th }}$ layer matrix, given by:

$\mathrm{C}_{\mathrm{r}}=\left[\begin{array}{cc}\mathrm{C}_{11} & \mathrm{C}_{12} \\ \mathrm{C}_{21} & \mathrm{C}_{22}\end{array}\right]=\left[\begin{array}{cc}\operatorname{Cos} \alpha_{\mathrm{r}} & -\mathrm{i} \sin \alpha_{\mathrm{r}} / \mathrm{q}_{\mathrm{r}} \\ -\mathrm{iq_{ \textrm {k } }} \sin \alpha_{\mathrm{r}} & \cos \alpha_{\mathrm{r}}\end{array}\right]$

Here $\alpha_{r}$ is the optical admittance given by,

$\alpha_{\mathrm{r}}=\mathrm{d}_{\mathrm{r}} \Upsilon_{0} \sqrt{\left(\epsilon_{\mathrm{r}}-\mathrm{n}^{2} \sin ^{2} \theta\right)}$

and phase factor, $\mathrm{q}_{\mathrm{k}}=\sqrt{\left(\epsilon_{\mathrm{r}}-\mathrm{n}^{2} \sin ^{2} \theta\right)} / \epsilon_{\mathrm{r}}$

where $\theta$ and $\Upsilon_{0}$ represents angle of incidence and wave number (free space).

After mathematical calculations, the value of reflectance for SPR sensor design (N layer) is given by [30]:

$R_{p}=\left|\frac{\left(C_{11}+C_{12} q_{n}\right) q_{p}-\left(C_{21}+C_{22} q_{n}\right)}{\left(C_{11}+C_{12} q_{n}\right) q_{p}+\left(C_{21}+C_{22} q_{n}\right)}\right|^{2}$

With the help of these equations, different performance parameters may be analyzed after plotting SPR curves [31].

(a) Sensitivity (S)

This parameter computes the sensing capability of the Bio-sensor, it is given as:

$\mathrm{S}=\frac{\Delta \theta_{\mathrm{SPR}}}{\delta \mathrm{n}_{\mathrm{s}}} \operatorname{deg}(\mathrm{RIU})^{-1}$

Where $\Delta \theta_{\text {SPR }}$ is the alteration in SPR resonance angle with change in sensing medium's $\mathrm{RI}\left(\delta \mathrm{n}_{\mathrm{s}}\right)$.

(b) Full-Width half maximum (FWHM)

The thickness of SPR curve at half (50\%) of reflectivity, given as

$F W H M=\theta_{\mathrm{b}}-\theta_{\mathrm{a}} \operatorname{deg}$ 
(c) Detection Accuracy (DA)

This parameter computes the exactness of the sensor and is expressed as:

$\mathrm{DA}=(\mathrm{FWHM})^{-1} \mathrm{Deg}^{-1}$

(d) Quality factor (Q) or Figure of merit (FoM)

Its mathematical expression is given by:

$$
F O M=S * D A R I U^{-1} \text {. }
$$

\subsection{Experimental Feasibility}

The feasibility of our proposed work can be proved with a sensor chip implementation and doing numerical simulation. To begin with, this part of the proposed work gives fabrication steps of metal, zinc sulfide $(\mathrm{ZnS})$, and graphene films, which finally design the SPR chips. The prism used here should be dipped in acetone vapor, methanol, and deionized water before being coupled to the metal layer. Over the BK7 glass, the physical vapor deposition of metal is done by a thermal evaporator system [32]. The fabrication of Graphene film can be done using a wellknown Chemical vapor deposition (CVD) method; further, it can be chemically moved above the $\mathrm{ZnS}$ sheet [33].

At last, these chips, after fabrication placed over BK7 glass and experimental values are calculated using a sensor setup [33], [34]. For sensing purposes, deionized water containing bacteria, viruses, as organic impurities are used. Further, this combination is poured over the SPR sensor chip for biomolecule and analyte sensing. Now to set up the angle of resonance, this whole combination puts it upon the goniometer having a rotary base shown in figure 1 . The input wave coming out of He-Ne laser source operating at a wavelength of $633 \mathrm{~nm}$, the coupling between p-polarized wave and BK7 prism done after passing light passes through different intermediate stages of the lens, polarizer, and collimator in between [33], [34]. On the other part of the prism, the reflected light gets detected by the optical photodetector that gives the output signal concerning the intensity of reflected light. The input laser light with an angle of incidence $(\theta)$ focusing on the surface of the sensor operating under the motor-based rotatory stage and it's controlling is with the help of computer's software. At last, the graphical plot of the reflectance curve can be plotted. 


\section{Results}

A low RI-based BK7 coupling prism is generally used for targeting high sensitivity SPR sensors [36]. While comparing the low RI-based prism with the high RI-based one, the high RI-based gives sharp resonance curves than the other. The parameters that define the characteristics of SPR sensors like FWHM, Sensitivity, SPR angle, and angular shift, their values are more significant in low RI-based glass than high RI-based glass [35].

A metal (Ag) film of $50 \mathrm{~nm}$ is placed upon a prism to get better values of the figure of merit and sharp resonance curve. By how much sensitivity gets improved, it is studied by placing zinc sulfide film on top of the metal film. On top of the zinc sulfide layer to enhance the analyte's adsorption in the sensor.

The impact of thickness variation of Ag layer on reflectance is shown in figure 2. The reflectance of the sensor varies with the thickness of the Ag metal layer. So, Ag metal's thickness needs to be optimized. It varies from $30 \mathrm{~nm}$ to $55 \mathrm{~nm}$. At $50 \mathrm{~nm}$ thickness of the $\mathrm{Ag}$ metal, the minimum reflectance 0.00559 is obtained, so Ag thickness equal to $50 \mathrm{~nm}$ is considered. It should be noted that the value of RI of the sensing layer is constant at 1.33.

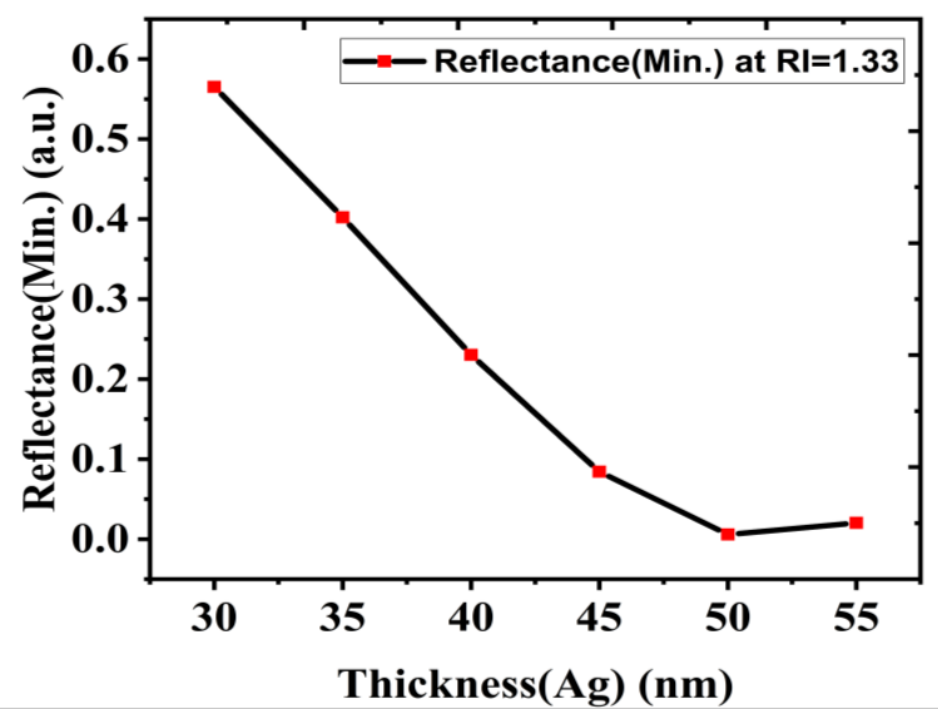

Figure 2: Minimum reflectance vs. Ag metal thickness plot at $1.33 \mathrm{RI}$

The sensitivity of the sensor also depends on the thickness of the dielectric material. Now the thickness of the $\mathrm{ZnS}$ and graphene vary at $1.33 \mathrm{RI}$. The first case is shown in figure 3(a) with 
$P=G=0$, i.e., it gives the graph for the conventional sensor. The SPR excitation proves to be there with the help of a sharp dip in reflectance curves, with the absorption of incident light falling on the prism-based sensor to produce SPs. The change in the resonance angle at $1.33 \mathrm{RI}$ is obtained as $1.165^{\circ}$, and the sensor's sensitivity is $116.5^{\circ} / R I U$ is achieved. The value for change in SPR resonance angle is $1.165^{\circ}$ in this case, i.e., $\delta \theta=1.165^{\circ}$ with a sensitivity of $116.5^{\circ}$ \% $R I U$ is obtained. Keeping other parameters constant, a graphene layer is added to the traditional sensor with no change in the zinc sulfide layer (i.e., $P=0, G=1$ ). From figure $3(\mathrm{~b})$, a further sharp dip in the resonance curve is seen with improving the sensitivity to $119^{\circ} / R I U$ with $\delta \theta=$ $1.19^{\circ}$. The enhancement in the sensitivity is due to the large excursion at the resonance curve. Also, the change in the resonance angle is increased. So it can be seen that with this case, both parameters are improved when compared with the traditional one, i.e., $P=0, G=0$. These improvements in the values are mainly due to the large cross-sectional area giving more significant adsorption given by graphene. These properties make it suitable for its applicability as a top layer dielectric in SPR sensor designing.

Figure 3(c) now explores the impact of adding a single zinc sulfide layer with no graphene layer on design parameters like sensitivity and $\delta \theta$. The change in the SPR angle and sensitivity further improve to $1.29^{\circ}$ and $129^{\circ} / R I U$, respectively. These values are better than both earlier cases. The values obtained here are due to zinc sulfide's optical properties like low bandgap with good adsorption efficiency. The last case shown by figure 3(d), consisting of a single layer of both materials (zinc sulfide and graphene), i.e., $P=1$ and $G=1$ shows further improvement in the parameters discussed earlier.

Further resonance shift and sensitivity achieved are $\delta \theta=1.327$ and sensitivity as $S=$ $132.7^{\circ} / R I U$. So, the best scenario is the last one which consists of both materials single layer. In addition to these cases, further, increase in the number of layers of zinc sulfide $(P \geq 2)$ and graphene as $(G \geq 2)$ the curves show growth in reflectance as shown in figure 5. From figure 5, it is clear that analysis of resonance angle is quite challenging to analyze. 


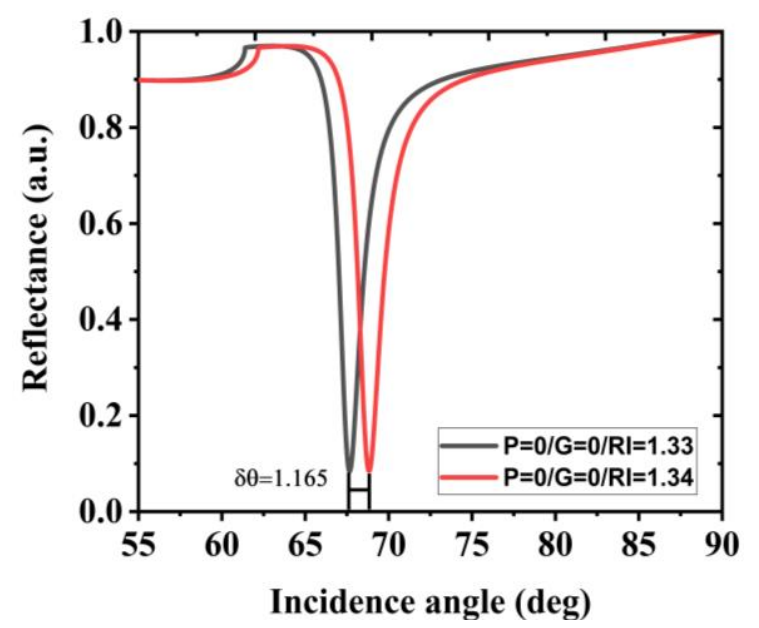

(a)

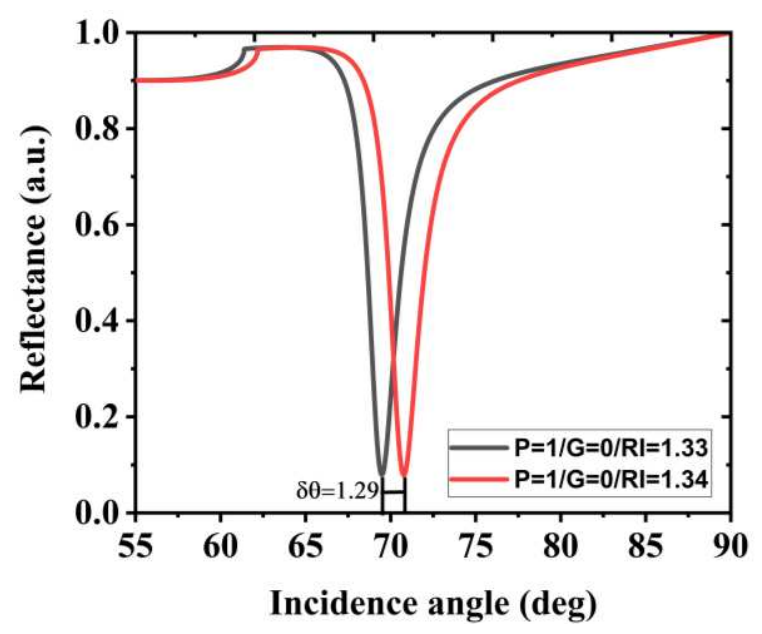

(c)

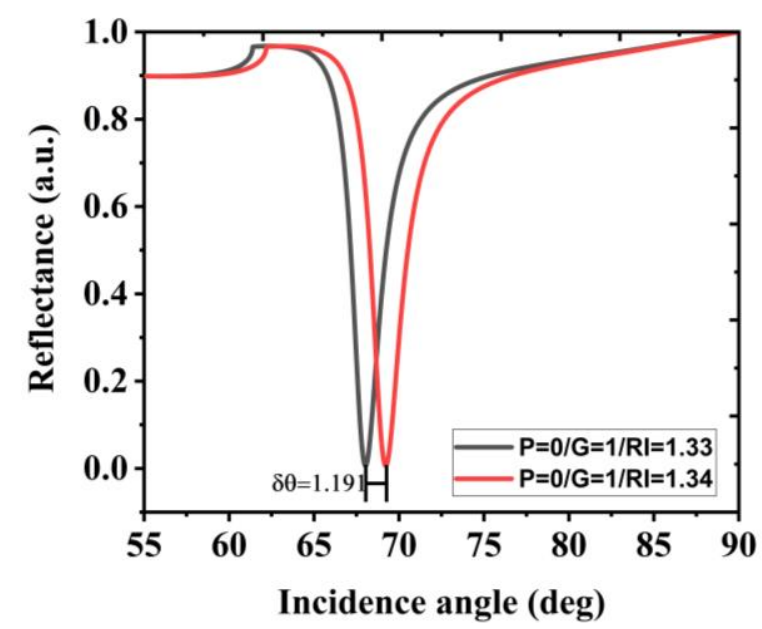

(b)

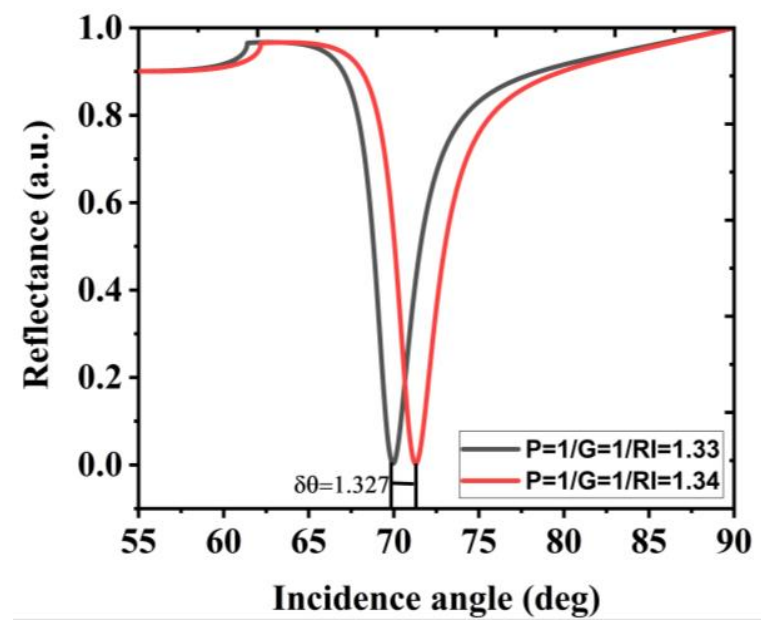

(d)

Figure 3 (a-d): Reflectance vs Incident angle plots for (a): $P / G=0,(\mathrm{~b}): P=0 / G=1$

$$
\text { (c): } P=1 / G=0(\mathrm{~d}): P=G=1
$$

The coming figure (4) gives the resulting plot for sensitivity concerning variation in RI of sensing layer (1.340 to 1.370 ). The sensitivity value shows the maximum numeric figure for a single film of $\mathrm{ZnS}$ and graphene $(P / G=1)$ in the range of $132.7^{\circ} / R I U$ to $168^{\circ} / R I U$ as compared to conventional combination $(P / G=0)$ which is showing $116.6^{\circ} / R I U$ to $138^{\circ} / R I U$. The following case $(P=0 / G=1)$, which is the modifications over traditional design, is the inclusion of a single graphene layer with no $\mathrm{ZnS}$ layer indicating sensitivity as $119^{\circ} / R I U$ (lowest) to $141.5^{\circ} / R I U$ (highest). Finally, for $(P=1 / G=0)$, the range achieved by sensitivity is $129-161^{\circ} / R I U$. 


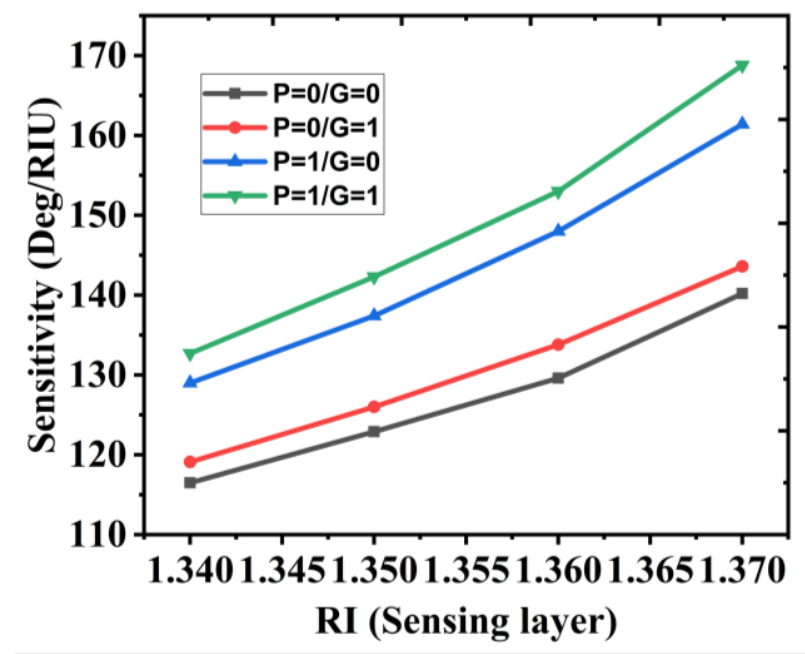

Figure 4: Variation in the sensitivity of the sensor by varying the RI of the sensing layer

This analysis depicts that the inclusion of $\mathrm{ZnS}$ and graphene layers in the proposed SPR biosensor increases the sensitivity compared with conventional cases $(P=0 / G=0)$. This statement further signifies that the reflectance increases by including different layers of graphene in the SPR sensor, and the SPR curve also broadens [figure 5(a)]. In the same way, adding further zinc sulfide nanosheets with a single graphene layer $(G=1 / P=$ varies $)$ its impact is shown in figure 5 (b) plot. The result shows a shift in reflectance dip, making SPR curve broader with the increase of graphene layers as 1 through 5.

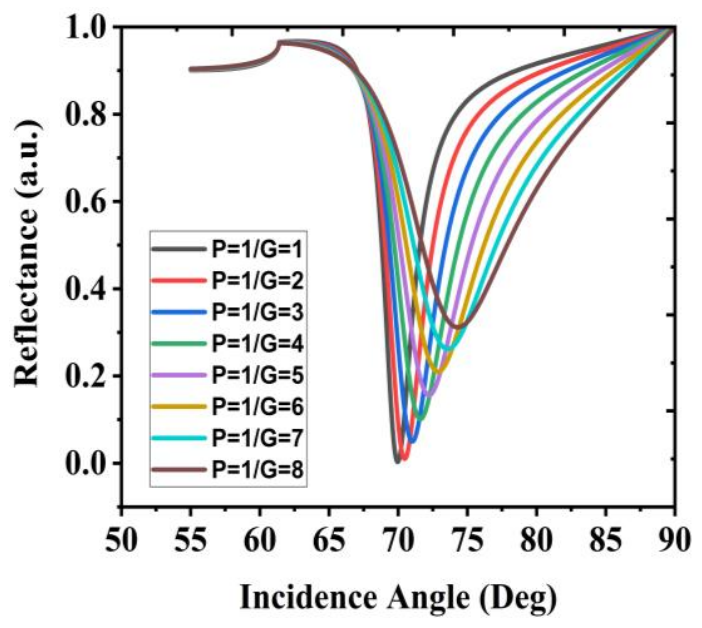

(a)

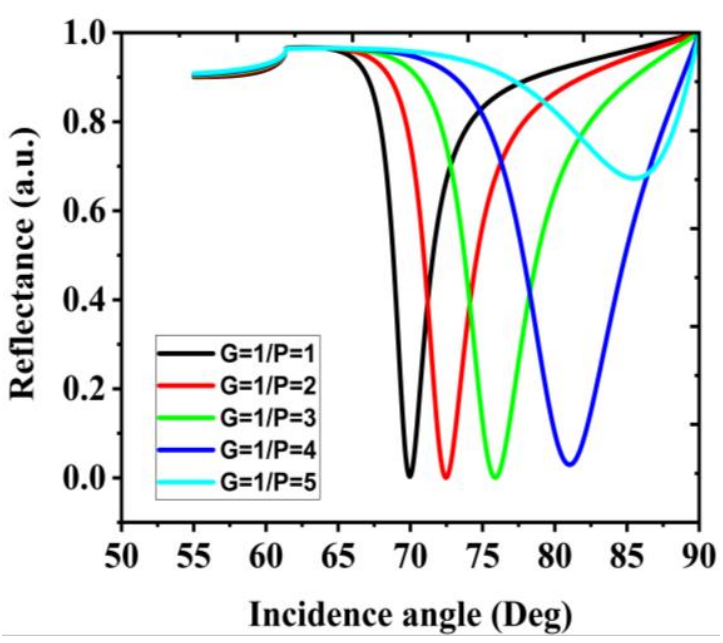

(b)

Figure 5: Different curves indicating the impact of incidence angle on reflectance (a) For $P=$ 1 (constant) and $G=$ varies ( 1 to 8 ) and (b) For $G=1$ (constant) and $P=$ varies ( 1 to 5 ) 
When the resonance curve broadens, the value of the resonance angle is difficult to get. The tabular analysis of various parameters with the structure type is shown in Table 2. The graphical realization for table 2 is represented in figure 6.

Table 2: Different parameters optimized values with different structures

\begin{tabular}{|c|c|c|c|c|c|}
\hline Structure & SPR angle & FWHM & Sensitivity & DA & $\mathbf{Q}$ \\
\hline (1) Ag/Prism & 67.655 & 1.165 & 116.5 & 0.86 & 100.2 \\
\hline (2) $\mathrm{Ag} / \mathrm{ZnS} /$ Prism & 69.479 & 1.29 & 129 & 0.78 & 100.6 \\
\hline (3) $\mathrm{Ag} / \mathrm{ZnS} /$ Graphene & 69.952 & 1.327 & 132.7 & 0.75 & 99.5 \\
\hline
\end{tabular}

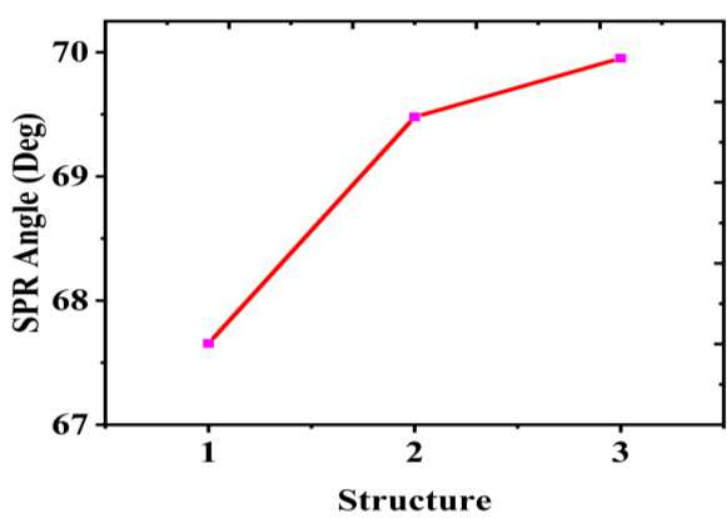

(a)

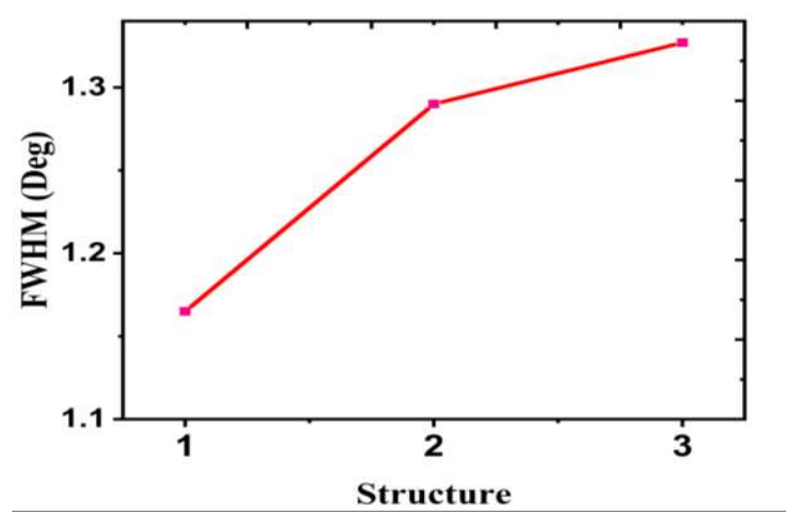

(b)

Figure 6 ( $a$ and $b$ ): SPR angle and FWHM curves for the structure 1, 2, 3

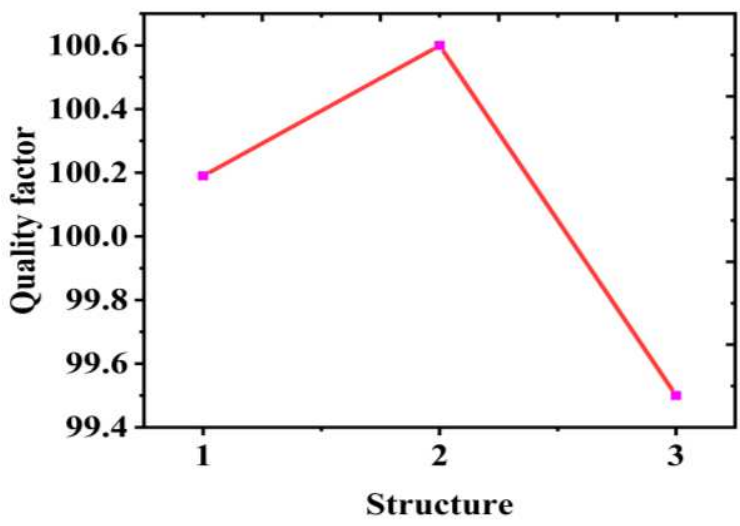

(c)

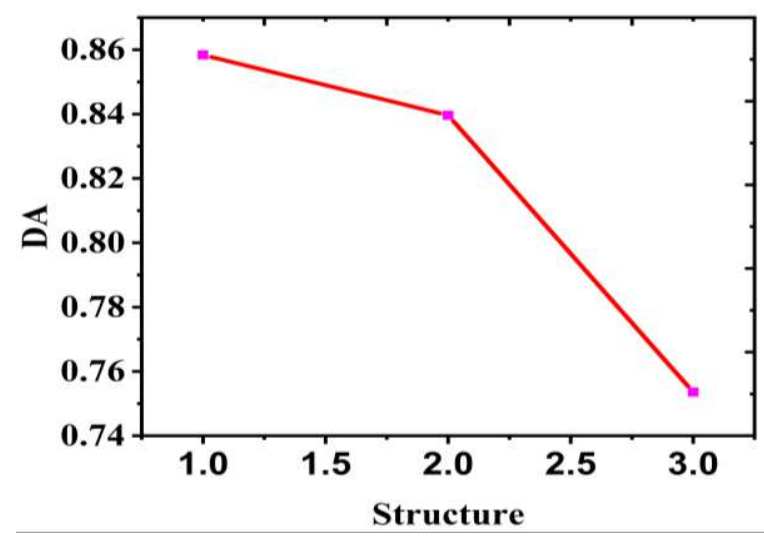

(d)

Figure 6 (c and d): Quality factor and DA curves for the structure 1, 2, 3 


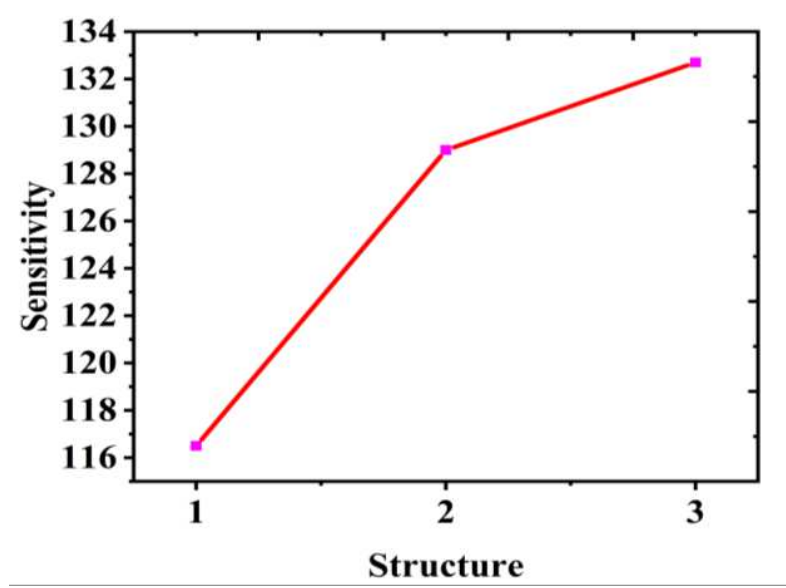

(e)

Figure 6 (e): Sensitivity for the structure 1, 2, 3

The upcoming table 3 shows the comparative parameter analysis with the previous research, clearly showing our proposed work achieves upgraded sensitivity compared to others.

Table 3: a comparative analysis of the proposed work with the previous literature

\begin{tabular}{|c|c|c|c|c|c|c|}
\hline References & Material used + BK7 prism & Operating $\lambda$ & $\mathrm{S}$ & DA & Q & FWHM \\
\hline$[31]$ & $\mathrm{Au} / \mathrm{BlueP} / \mathrm{MoS} 2$ Antimonene & $633 \times 10^{-9} \mathrm{~m}$ & 194.8 & 0.152 & - & 6.60 \\
\hline$[19]$ & $\mathrm{Ag} / \mathrm{Au} / \mathrm{BaTiO}_{3 / \text { graphene }}$ & $633 \times 10^{-9} \mathrm{~m}$ & 294 & & 42.13 & 6.978 \\
\hline$[12]$ & $\mathrm{Au} / \mathrm{MoS}_{2} / \mathrm{Ni} / \mathrm{Graphene}$ & $633 \times 10^{-9} \mathrm{~m}$ & 229 & - & - & - \\
\hline$[36]$ & $\mathrm{ZnO} / \mathrm{Ag} / \mathrm{Au} /$ graphene & $632.8 \times 10^{-9} \mathrm{~m}$ & 76 & - & 13.79 & 5.510 \\
\hline This work & $\mathrm{Ag} / \mathrm{ZnS} / \mathrm{Graphene}$ & $633 \times 10^{-9} \mathrm{~m}$ & 305 & 0.33 & 100.7 & 3.05 \\
\hline
\end{tabular}

\section{Conclusion}

The proposed work explains the theoretical performance analysis of SPR sensor with the computation of different parameters. The design of the sensor consists of the zinc sulfide layer mounted on the Ag layer. The graphene as 2D material is used to increase the sensitivity of the sensor. The maximum sensitivity of the sensor is achieved $305^{\circ} / \mathrm{RIU}$ with four $\mathrm{ZnS}$ and single graphene layer. The optimized metal layer thickness was taken as $50 \mathrm{~nm}$. The whole study uses a $\mathrm{P}$ polarized monochromatic He-Ne input source, operating at $633 \mathrm{~nm}$. The detection accuracy, quality factor, and FWHM is obtained as $0.33 \mathrm{deg}^{-1}, 100.7 \mathrm{RIU}^{-1}$ and $3.05 \mathrm{deg}$ respectively. 
The proposed sensor design might be helpful in different medical, environmental, chemical studies.

Code Availability: Not applicable.

Funding Information: No funding available.

Conflicts of interest/Competing interests: The author declare that he has no conflict of interest.

Availability of data and material: No data available.

\section{Authors' contributions}

BK formulated the problem statement wherein giving the theoretical background and mathematical modelling for SPR biosensor. He also helped in drafting and finalizing the manuscript.

AU provided the theoretical background to biosensing and the importance of Optical Biosensing. He also helped in finalizing the design of the proposed sensor.

BLC worked towards the complete manuscript, formatting, and finalizing the manuscript.

AP provided statistical analysis for the results. He provided the theoretical background to SPR biosensors. He also helped in formatting the manuscript.

\section{Ethics approval}

Not applicable. The work presented in this manuscript is mathematical modelling only for the proposed biosensor. No experiment was performed on the human body and/or living organism/ animal. So, an ethical approval from an ethical committee is not required.

\section{Consent to participate}

I am willing to participate in the work presented in this manuscript.

\section{Consent for publication}

The author has given their consent to publish this work.

\section{References}

[1] H. C. Lee, C. T. Li, H.-F. Chen, and T.-J. Yen, "Demonstration of an ultrasensitive refractive-index plasmonic sensor by enabling its quadrupole resonance in phase interrogation," Opt. Lett., vol. 40, no. 22, p. 
5152, 2015.

[2] G. Zheng, Y. Chen, L. Bu, L. Xu, and W. Su, "Waveguide-coupled surface phonon resonance sensors with super-resolution in the mid-infrared region," Opt. Lett., vol. 41, no. 7, p. 1582, 2016.

[3] E. Ozbay, "Plasmonics: Merging photonics and electronics at nanoscale dimensions," Science (80-. )., vol. 311, no. 5758, pp. 189-193, 2006.

[4] S. K. Srivastava, R. Verma, and B. D. Gupta, "Theoretical modeling of a self-referenced dual mode SPR sensor utilizing indium tin oxide film," Opt. Commun., vol. 369, pp. 131-137, 2016.

[5] B. Liedberg, C. Nylander, and I. Lunström, "Surface plasmon resonance for gas detection and biosensing," Sensors and Actuators, vol. 4, pp. 299-304, 1983.

[6] M. Chamtouri, M. Sarkar, J. Moreau, M. Besbes, H. Ghalila, and M. Canva, "Field enhancement and target localization impact on the biosensitivity of nanostructured plasmonic sensors," J. Opt. Soc. Am. B, vol. 31, no. 5, p. 1223, 2014.

[7] H. Vahed and C. Nadri, "Sensitivity enhancement of SPR optical biosensor based on Graphene-MoS2 structure with nanocomposite layer," Opt. Mater. (Amst)., vol. 88, pp. 161-166, 2019.

[8] J. Homola, S. S. Yee, and G. Gauglitz, "Surface plasmon resonance sensors: review," Sensors Actuators, $B$ Chem., vol. 54, no. 1, pp. 3-15, 1999.

[9] J. Homola, "Present and future of surface plasmon resonance biosensors," Anal. Bioanal. Chem., vol. 377, no. 3, pp. 528-539, 2003.

[10] M. K. Singh, S. Pal, A. Verma, V. Mishra, and Y. K. Prajapati, "Sensitivity enhancement using anisotropic black phosphorus and antimonene in bi-metal layer-based surface plasmon resonance biosensor," Superlattices Microstruct., vol. 156, p. 106969, 2021.

[11] A. Pal and A. Jha, "A theoretical analysis on sensitivity improvement of an SPR refractive index sensor with graphene and barium titanate nanosheets," Optik (Stuttg)., vol. 231, p. 166378, 2021.

[12] A. Nisha, P. Maheswari, P. M. Anbarasan, K. B. Rajesh, and Z. Jaroszewicz, "Sensitivity enhancement of surface plasmon resonance sensor with $2 \mathrm{D}$ material covered noble and magnetic material (Ni)," Opt. Quantum Electron., vol. 51, no. 19, 2019.

[13] A. Otto, "Excitation of nonradiative surface plasma waves in silver by the method of frustrated total reflection," Zeitschrift für Phys., vol. 216, no. 4, pp. 398-410, 1968.

[14] E. Kretschmann and H. Raether, "Radiative Decay of Non Radiative Surface Plasmons Excited by Light," Zeitschrift fur Naturforsch. - Sect. A J. Phys. Sci., vol. 23, no. 12, pp. 2135-2136, 1968.

[15] D. Cai, Y. Lu, K. Lin, P. Wang, and H. Ming, "Improving the sensitivity of SPR sensors based on gratings by double-dips method (DDM)," Opt. Express, vol. 16, no. 19, p. 14597, 2008.

[16] M. Lee, H. Jeon, and S. Kim, "A highly tunable and fully biocompatible silk nanoplasmonic optical sensor," Nano Lett., vol. 15, no. 5, pp. 3358-3363, 2015.

[17] M. M. Rahman, M. M. Rana, M. S. Rahman, M. S. Anower, M. A. Mollah, and A. K. Paul, "Sensitivity enhancement of SPR biosensors employing heterostructure of PtSe2 and 2D materials," Opt. Mater. (Amst)., vol. 107, p. 110123, 2020.

[18] L. Wu, H. S. Chu, W. S. Koh, and E. P. Li, "Highly sensitive graphene biosensors based on surface plasmon resonance," Opt. Express, vol. 18, no. 14, p. 14395, 2010.

[19] S. Chen and C. Lin, "Sensitivity comparison of graphene based surface plasmon resonance biosensor with $\mathrm{Au}, \mathrm{Ag}$ and $\mathrm{Cu}$ in the visible region," Mater. Res. Express, vol. 6, no. 5, pp. 1108-1116, 2019.

[20] G. Alaguvibisha et al., "Sensitivity enhancement of surface plasmon resonance sensor using hybrid configuration of 2D materials over bimetallic layer of Cu-Ni," Opt. Commun., vol. 463, p. 125337, 2020.

[21] X. Wang, Z. Xie, H. Huang, Z. Liu, D. Chen, and G. Shen, "Gas sensors, thermistor and photodetector based on ZnS nanowires," J. Mater. Chem., vol. 22, no. 14, pp. 6845-6850, 2012.

[22] Y. Singh and S. K. Raghuwanshi, "Electromagnetic wave sensors Sensitivity Enhancement of the Surface Plasmon Resonance Gas Sensor With Black Phosphorus," IEEE Sensors Lett., vol. 3, no. 12, pp. 1-4, 2019.

[23] G. S. Mei, P. Susthitha Menon, and G. Hegde, "ZnO for performance enhancement of surface plasmon resonance biosensor: A review," Mater. Res. Express, vol. 7, no. 1, p. 012003, 2020.

[24] Y. Singh, M. K. Paswan, and S. K. Raghuwanshi, "Sensitivity Enhancement of SPR Sensor with the Black Phosphorus and Graphene with Bi-layer of Gold for Chemical Sensing," Plasmonics, 2021.

[25] M. Pumera, "Graphene in biosensing," Mater. Today, vol. 14, no. 7-8, pp. 308-315, 2011.

[26] B. Karki, S. Sharma, Y. Singh, and A. Pal, "Sensitivity Enhancement of Surface Plasmon Resonance Biosensor with 2-D Franckeite Nanosheets," Plasmonics, pp. 1-16, 2021.

[27] Q. Ouyang et al., "Sensitivity Enhancement of Transition Metal Dichalcogenides/Silicon Nanostructurebased Surface Plasmon Resonance Biosensor," Sci. Rep., vol. 6, no. June, pp. 1-13, 2016. 
[28] S. Gan, Y. Zhao, X. Dai, and Y. Xiang, "Sensitivity enhancement of surface plasmon resonance sensors with 2D franckeite nanosheets," Results Phys., vol. 13, p. 102320, 2019.

[29] A. Srivastava and Y. K. Prajapati, "Performance Analysis of Silicon and Blue Phosphorene / MoS 2 HeteroStructure Based SPR Sensor,” PHOTONIC SENSORS, vol. 9, no. 3, pp. 284-292, 2019.

[30] R. Jha and A. K. Sharma, "Chalcogenide glass prism based SPR sensor with Ag-Au bimetallic nanoparticle alloy in infrared wavelength region," J. Opt. A Pure Appl. Opt., vol. 11, no. 4, 2009.

[31] M. K. Singh, S. Pal, Y. K. Prajapati, and J. P. Saini, "Sensitivity Improvement of Surface Plasmon Resonance Sensor on Using BlueP/MoS 2 Heterostructure and Antimonene," IEEE Sensors Lett., vol. 4, no. 7, 2020.

[32] S. Agarwal, Y. K. Prajapati, and J. B. Maurya, "Effect of metallic adhesion layer thickness on surface roughness for sensing application," IEEE Photonics Technol. Lett., vol. 28, no. 21, pp. 2415-2418, 2016.

[33] P. Chen, N. Li, X. Chen, W. J. Ong, and X. Zhao, "The rising star of 2D black phosphorus beyond graphene: Synthesis, properties and electronic applications," 2D Mater., vol. 5, p. 014002, 2018.

[34] J. B. Maurya and Y. K. Prajapati, "Experimental Demonstration of DNA Hybridization Using Graphene Based Plasmonic Sensor Chip," J. Light. Technol., vol. 38, no. 18, pp. 5191-5198, 2020.

[35] K. Brahmachari and M. Ray, "Effect of prism material on design of surface plasmon resonance sensor by admittance loci method," Front. Optoelectron., vol. 6, no. 2, pp. 185-193, 2013.

[36] R. Kumar, A. S. Kushwaha, M. Srivastava, and H. M. S. K. Srivastava, "Enhancement in sensitivity of graphene-based zinc oxide assisted bimetallic surface plasmon resonance ( SPR ) biosensor," Appl. Phys. A, vol. 124 , no. 235 , p. $0,2018$. 